\title{
Reviewer acknowledgement Mammalia volume 82 (2018)
}

https://doi.org/10.1515/mammalia-2018-0168

The editors and the publisher wish to thank the following colleagues for their kind assistance in acting as referees for the journal this year: ${ }^{1}$

\author{
Abba, Agustin M. \\ Adamik, Peter \\ Adler, Gregory \\ Aguilera, Marisol \\ Álvarez-Castañeda, Sergio \\ Anacleto, Teresa Cristina \\ Ancillotto, Leonardo \\ Araujo, Ana Carolina \\ Aryal, Achyut \\ Astúa, Diego \\ Aymerich, Pere \\ Bacon, Michelle \\ Bagchi, Sumanta \\ Baillieul, John \\ Bakloushinskaya, Irina \\ Baldwin, Roger \\ Barbanti Duarte, Jose Mauricio \\ Barbaro, Luc \\ Barbier, Eder \\ Bar-Gal, Gila Kahila \\ Barrientos, Rafael \\ Beck, Harald \\ Beisiegel, Beatriz \\ Benson, John \\ Bertassoni, Alessandra \\ Bertolino, Sandro \\ Bianchi, Rita de Cassia \\ Bidau, Claudio \\ Biffi, Marjorie \\ Bilgin, Rasit \\ Biondo, Cibele \\ Birks, Johnny \\ Bogdanowicz, Wielsaw \\ Bogdziewicz, Michal \\ Bogoni, Juliano \\ Boonman, Arjan \\ Boratynski, Zbyszek \\ Bounaceur, Farid \\ Bradley, Robert \\ Brandon-Jones, Douglas \\ Brito, Jorge \\ Bryja, Josef \\ Butet, Alain
}

Camps, David

Canel, Delfina

Cantor, Mauricio

Carraway, Leslie

Castelblanco, Nataly

Ceia, Ricardo

Cerna Bolfikova, Barbora

Chaiyarat, Rattanawat

Chang, Shih-Wei

Chevret, Pascale

Ciechanowski, Mateusz

Clemente-Sanchez, Fernando

Cotza, Antonella

Cove, Mike

Croose, Elizabeth

Cuzin, Fabrice

David, Léa

de Carvalho Falcão, Fábio

de la Torre, J. Antonio

de Moura, Jailson Fulgencio

Decher, Jan

Deguchi, Yoshitaka

Delciellos, Ana

D’Elía, Guillermo

Delsuc, Frederic

Desbiez, Arnaud

Diggins, Corinne

Dinets, Vladimir

Doss, Paramanantha Swami D.

Drouilly, Marine

du Preez, Byron

Fellous, Amina

Feng, Jiang

Fernández, Jesús

Ferreguetti, Atilla

Ferreira, Diogo

Fuller, Todd

Ganem, Guila

Gannier, Alexandre

Garbino, Guilherme

García, Franger J.
Gardner, Alfred L.

Garg, S.K.

Ghosh, Sonali

Giraudoux, Patrick

Gol'din, Pavel

Gomes Rodrigues, Helder

Govindan Veeraswami, Gopi

Gowans, Shannon

Graipel, Mauricio

Grassman, Lon

Greene, Daniel

Gregorin, Renato

Grueter, Cyril

Guimarães, Bruno

Guntiñas, Marta

Haberl, Werner

Habib, Bilal

Hackett, Talya

Haigh, Amy

Hedges, Laurie

Hendershott, Rebecca

Hernández-Betancourt, Silvia

Hope, Andrew

Hussain, Syed

Hutterer, Rainer

Ivajnsic, Danijel

Janzekovic, Franc

Jenkins, Paulina

Jiang, Xuelong

Johnsingh, A.J.T.

Jordan, Christopher

Juškaitis, Rimvydas

Juste, Javier

Kalle, Riddhika

Kam, Michael

Kambali, Kamarul Ariffin

Kamjing, Anucha

Kanuch, Peter

Katona, Krisztian

Kerbis Peterhans, Julian

1 The copy deadline for the list was October 9, 2018. 
Kettel, Esther

Kishimoto, Ryosuke

Kitchener, Andrew

Kittle, Andrew

Kolipaka, Srinivas Shekhar

Koprowski, John

Koubinova, Darina

Kowalewski, Martin

La Haye, M.J.J.

Lambert, Mark

Lanszki, Jozsef

Leiner, Natalia

Leite de Oliveira, Márcio

Libois, Roland

Limoilou, Renaud

Long, Eric

Long, Yong-Chen

Lopez-Aguirre, Camilo

Lopez-Gonzalez, Celia

Loretto, Diogo

Lovari, Sandro

Lucati, Federica

Mahmoudi, Ahmad

Maldonado, Jesús

Mallon, David

Mann, Gareth

Mares, Michael

Marin, Gabriela

Martinkova, Natalia

Matos, Milene

McCarthy, Jennifer

Mcshea, William

Mead, James G.

Merceron, Gildas

Merou, Theodora

Mikula, Peter

Milne, Damian J.

Moe, Stein

Mohd-Azlan, Jayasilan

Molinari, Jesus

Monadjem, Ara

Monecke, Stefanie

Montuire, Sophie

Mora, Jose

Morais, Igor

Mori, Emiliano

Morita, Tetsuo

Morni, Muhd Amsyari

Morris, Patric

Muylaert, Renata

Nadachowski, Adam

Nadeem, Muhammad

Nathan, P. Thiruchenthil

Neumann, Wiebke

Newsome, Thomas

Ngoprasert, Dusit
Nicolas, Violaine

Nkrumah, Evans E.

Norris, Darren

Novaes, Roberto Leonan

Novák, Peter

Novillo, Agustina

Novotny, Frantsek

Oliveira, Leonardo C.

Olsen, Morten Tange

Olson, Kirk

Ortíz, Pablo

Ossa, Gonzalo

Palomares, Francisco

Pantoja, Tatyana Mariucha

Patterson, Bruce

Pavlinić, Igor

Pedersen, Scott C.

Penteriani, Vincent

Pérez-Irineo, Gabriela

Perrin, Mike

Phalan, Ben

Phillipps, Quentin

Postawa, $\mathrm{T}$.

Prado, Joyce

Prevedello, Jayme

Pudyatmoko, Satyawan

Puechmaille, Sébastien

Qihai, Zhou

Queirolo, Diego

Quere, Jean-Pierre

Querejeta, Marina

Racey, Paul

Rapaport, Lisa G.

Rathbun, Galen

Reiners, Tobias Erik

Reyes, Adriana

Reza, AHM

Rödel, Heiko

Rodrigues Bonvicino, Cibele

Rodriguez, Alejandro

Rogovin, Konstantin

Rose, Andreas

Ruedi, Manuel

Ruelas, Dennisse

Rychlik, Leszek

Ryser, Marie Pierre

Saldaña-Vázquez, Romeo A.

Salles, Leandro

Sánchez Hernández, Cornelio

Santori, Ricardo

Santos-Moreno, Antonio

Schiaffini, Mauro

Schroeder, Natalia

Scott, Dawn M.
Sedláček, František

Self, Casey J.

Semedo, Thiago

Serrano, Emmanuel

Sethy, Janmejay

Shapira, Idan

Sharma, Hari

Sharma, Koustubh

Shrestha, Rinjan

Siciliano, Salvatore

Silva, Luis

Simonetti, Javier

Singh, Mewa

Singh, Randeep

Soisook, Pipat

Souza, Renan

Srinivasulu, Chelmala

Steen, Ronny

Steinmetz, Robert

Stephenson, P.J.

Stoetzel, Emmanuelle

Sumbera, Radim

Sun, Keping

Teta, Pablo

Thevenot, Michel

Thibault, Martin

Tinoco Lopez, Nicolas

Tobler, Mathias

Torre, Ignasi

Trujano-Alvarez, Ana

Tzanopoulos, Joseph

Udrizar Sauthier, Daniel

Uhrin, Marcel

Urbàn R., Jorge

Van Vuren, Dirk H.

Vargas-Mena, Juan Carlos

Varzinczak, Luiz

Velazco, Paúl

Voss, Robert

Wang, Yong

Wechuli, David B.

Weinberger, Irene

Weldone, Andy

Willcox, Daniel

Wilson, Cara

Winkler, Daniela

Xiang, Zuofu

Zachos, Frank E.

Zahler, Peter

Zahn, Andreas

Zuberogoitia, Inigo

Zurano, Juan Pablo 